\title{
On cycles in models of asymmetric circular gene networks
}

\author{
V. Golubyatnikov ${ }^{1,2 *}$, N. Kirillova ${ }^{2}$ \\ ${ }^{1}$ Sobolev Institute of Mathematics SB RAS, Novosibirsk, Russia \\ ${ }^{2}$ Novosibirsk State University, Novosibirsk, Russia \\ *e-mail: vladimir.golubyatnikov1@fulbrightmail.org
}

Key words: Circular gene networks, equilibrium points, cycles

Motivation and Aim: We consider nonlinear dynamical systems as models of functioning of asymmetric circular gene networks more complicated and general than analogous models studied in [1-3]. Our main aim here is to find conditions of existence of oscillating trajectories (cycles) of these systems.

Methods and Algorithms: Our constructions and studies of circular gene networks models and description of geometric and combinatorial structures of their phase portraits are based on our previous results, see [3]. In our numerical experiments we used the soft STEP elaborated in the Sobolev institute of mathematics.

Results: For positive parameters $k_{j}$ and $\mu_{s}$ and positive monotonically decreasing smooth functions $f_{m}, m=1,5,8$, which describes negative feedbacks in the gene network, we consider 9D-dynamical system

$\frac{d x_{1}}{d t}=f_{1}\left(x_{9}\right)-k_{1} x_{1} ; \frac{d x_{j}}{d t}=f_{j}\left(x_{j-1}\right)-k_{j} x_{j} ; j=5,8 ;$

$\frac{d x_{s}}{d t}=\mu_{s}\left(x_{s-1}\right)-k_{s} x_{s} ; s=2,3,4,6,7,9$.

Here $x_{1}, x_{5}, x_{8}$ are concentrations of mRNA's, and all the other variables denote concentrations of proteins which are "intermediate" stages of this gene network functioning. Here, in contrast with [1-3], several intermediate stages can appear between each pair of mRNA's with consecutive indices, not just one.

We show uniqueness of equilibrium point $S_{0}$ of the system (1) and find conditions of existence of a cycle $\boldsymbol{C}$ of this system, and describe an invariant polyhedral domain $W$ of this system in the positive octant of 9-D space and contains $\boldsymbol{C}$. These conditions are formulated in terms of matrix of linearization of the system (1) at the point $S_{0}$ : the nondiagonal non-zero terms of this matrix should be sufficiently large with respect to the parameters $k_{j}, k_{s}$. The invariant domain $W$ is composed by 18 adjacent parallelepipeds and retracts to $\boldsymbol{C}$. Our numerical experiments illustrate and correspond to the theoretical results. We show non-uniqueness of the cycles in some higher-dimensional dynamical systems of the type (1).

Conclusion: In contrast with [2], where the particular case $m_{1}=m_{2}=m_{1}=1$ symmetric with respect to cyclic permutations of the variables was studied, the shifts along trajectories of the system (1) are not described by equations with delayed arguments. The cycle $C$ is not symmetric with respect to this permutation.

Acknowledgements: Supported by RFBR (18-01-00057) and by complex program of basic research of SB RAS (0314-2018-0011).

\section{References}

1. Elowitz M.B., Leibler S. (2000) A Synthetic Oscillatory Network of Transcriptional Regulators. Nature, 335-338.

2. Kolesov A.Yu., Rozov N.Kh., Sadovnichii V.A. (2016) Periodic Solutions of Travelling-Wave Type in Circular Gene Networks. Izvestiya RAN: Ser. Mat. 80(3):67-94.

3. Ayupova N.B., Golubyatnikov V.P., Kazantsev M.V. (2017) On the Existence of a Cycle in an Asymmetric Model of a Molecular Repressilator. Numerical Analysis and Applications. 10(2):101-107. 